\title{
Accords transnationaux d'entreprise et dialogue social sectoriel européen
}

Quelles interactions?

Transnational company agreements and the European sectoral social dialogue

\section{Évelyne Léonard et André Sobczak}

\section{(Q) OpenEdition}

\section{Journals}

\section{Édition électronique}

URL : http://journals.openedition.org/travailemploi/1609

DOI : 10.4000/travailemploi.1609

ISSN : 1775-416X

\section{Éditeur}

DARES - Ministère du Travail

\section{Édition imprimée}

Date de publication : 15 mars 2010

Pagination : 43-54

ISSN : 0224-4365

\section{Référence électronique}

Évelyne Léonard et André Sobczak, « Accords transnationaux d'entreprise et dialogue social sectoriel européen », Travail et Emploi [En ligne], 121 | janvier-mars 2010, mis en ligne le 15 mars 2012, consulté le 30 avril 2019. URL : http://journals.openedition.org/travailemploi/1609 ; DOI : 10.4000/ travailemploi.1609 


\title{
Accords transnationaux d'entreprise et dialogue social sectoriel européen: quelles interactions?
}

\author{
Évelyne Léonard (*), André Sobczak (**)
}

\begin{abstract}
Alors que le dialogue social transnational se développe rapidement au niveau de l'entreprise et au niveau sectoriel, peu de recherches ont été conduites sur les liens qui peuvent exister entre ces deux niveaux. Cet article présente les principaux résultats d'une étude sur les interactions entre les différents niveaux de dialogue social transnational, en se concentrant sur celles qui relient les accords transnationaux d'entreprise et le dialogue social sectoriel européen. Il montre que de nombreux liens peuvent être observés entre ces niveaux de dialogue social transnational, qu'il s'agisse des acteurs impliqués, des thématiques abordées ou des procédures de mise en æuvre mobilisées. Certes, on est loin d'une articulation systématique et formalisée telle qu'elle existe dans certains systèmes nationaux, mais les liens que l'on peut observer indiquent des interdépendances croissantes qui peuvent contribuer à la construction progressive d'un système de relations professionnelles transnationales.
\end{abstract}

Au cours des vingt dernières années, le dialogue social transnational s'est fortement développé, surtout au niveau des entreprises et des secteurs (1), reflétant le caractère de plus en plus transnational des activités des entreprises et la volonté de certains acteurs d'établir une régulation sociale de celles-ci. Ce développement concerne avant tout le niveau européen, mais aussi, dans une moindre mesure, le niveau mondial.

Il se manifeste, en particulier, par l'accroissement du nombre d'accords transnationaux d'entreprise (Commission européenne, 2008a; Schoemann et al., 2008) et des activités des comités de dialogue social sectoriel au sein de l'Union européenne (Pochet et al., 2009). Le développement de ces deux formes de dialogue social transnational pose la question de leurs interactions, d'autant plus que ces pratiques ne s'inscrivent pas nécessairement dans un cadre juridique précis et une architecture établie de relations professionnelles, surtout dans le cas des textes négociés au niveau des entreprises.

Alors que peu de recherches abordent directement cette question, l'analyse des articulations entre

(*) Institut des sciences du travail, université catholique de Louvain, evelyne.leonard@uclouvain.be

(**) Institut pour la Responsabilité Globale dans l'Entreprise, Audencia Nantes School of Management, asobczak@audencia. com

(1) Plusieurs accords ont aussi été adoptés dans le cadre du dialogue social européen au niveau interprofessionnel (MIAS, 2004). En revanche, le dialogue social international, que ce soit au niveau sectoriel ou interprofessionnel, reste presque inexistant. les différents niveaux de dialogue social transnational présente un réel intérêt pour l'ensemble des acteurs concernés. Créer des liens entre les différents niveaux, favoriser des synergies ou évoquer des pistes pour répartir les rôles entre eux sont autant d'éléments qui permettraient de renforcer l'efficacité des deux niveaux du dialogue social transnational. Dans les différents contextes nationaux, la question de l'articulation entre niveaux du dialogue social fait d'ailleurs l'objet de recherches et de débats, voire souvent d'une régulation imposée par les pouvoirs publics ou négociée entre les partenaires $\operatorname{sociaux}(2)$.

Cet article se fixe pour objectif de contribuer à combler cette lacune et cherche à explorer les différents liens actuels et potentiels entre le dialogue social transnational au niveau du secteur et de l'entreprise, le premier étant presque toujours limité au cadre européen alors que le second inclut souvent une dimension plus internationale. L'article repose sur une recherche exploratoire menée pour la DARES entre juillet 2008 et janvier 2009, et fondée sur l'analyse documentaire des principaux textes négociés aux deux niveaux ainsi que sur des entretiens semi-directifs avec une vingtaine d'acteurs du dialogue social transnational et des experts. Il vise à illustrer que, contrairement à ce que l'absence de recherches sur ce sujet pourrait laisser entendre, les

(2) Ainsi, en France, les règles sur l'articulation entre les différents niveaux du dialogue social sont définies par la loi, alors qu'en Italie, elles ont fait l'objet d'une négociation entre les partenaires sociaux. 
interactions entre ces deux niveaux existent et ont tendance à se renforcer, offrant ainsi de nombreuses opportunités pour l'ensemble des partenaires sociaux impliqués.

Dans une première partie, 1'article établit un état des lieux très succinct de chacun de ces deux niveaux du dialogue social. La deuxième partie analyse les interactions entre ces niveaux, qu'il s'agisse des acteurs impliqués, des thématiques abordées et des procédures de mise en œuvre mobilisées. En conclusion, l'article cherche à esquisser certaines pistes pour l'avenir du dialogue social transnational et les interactions entre ces deux niveaux.

\section{Deux niveaux de dialogue social transnational en développement}

Avant d'analyser les interactions, il est nécessaire de présenter les grands traits des accords transnationaux d'entreprise et du dialogue social sectoriel européen. Il peut paraître curieux de se limiter à ces deux niveaux, en négligeant notamment le dialogue social interprofessionnel européen. La raison en est que ces deux niveaux ont connu les développements les plus importants au cours des quinze dernières années, mais aussi qu'ils posent des questions inédites sur l'émergence potentielle d'un système de relations professionnelles au-delà des frontières nationales et, plus spécifiquement sur les possibles interactions entre le niveau de l'entreprise et celui du secteur.

\section{L'essor des accords transnationaux d'entreprise: dynamiques internationales et européennes}

Apparus à la fin des années 1980, les accords transnationaux d'entreprise se sont multipliés à partir de 2000. Dans un document de travail publié en juillet 2008, la COMMISSION EUROPÉENNE (2008a) recense 147 textes négociés dans 89 entreprises. Parmi ces textes, 76 ont un champ d'application limité aux filiales situées sur le territoire de l'Union européenne, alors que 59 s'appliquent dans le monde entier et que 12 contiennent à la fois des dispositions ayant une vocation mondiale et des normes se limitant à l'Europe. La Commission ellemême considère que ce chiffre est sous-estimé, car certains accords transnationaux d'entreprise, surtout ceux qui ont une vocation européenne, ne sont pas diffusés par les acteurs qui les ont négociés.

Dans le domaine du dialogue social transnational au sein de l'entreprise, la distinction entre la sphère internationale et la sphère européenne peut être questionnée. En effet, même dans la sphère européenne, aucun cadre institutionnel ou juridique n'existe à ce jour pour les accords transnationaux d'entreprise (SoBCZAK, 2007), à part pour ceux, très spécifiques, qui portent sur la mise en place du comité d'entreprise européen(3) ou sur la place des salariés dans la société européenne(4). Cela place d'ailleurs ces textes dans un statut tout à fait particulier par comparaison aux textes du dialogue social européen de niveau interprofessionnel ou de niveau sectoriel, lesquels bénéficient d'un encadrement institutionnel précisément défini (voir à ce sujet, en particulier, DidRY, MiAs (2005), pour une analyse approfondie du dialogue social européen interprofessionnel; Pochet et al. (2009), pour une analyse du niveau sectoriel).

Par ailleurs, les accords transnationaux ayant une vocation mondiale sont, dans leur très grande majorité, négociés par des entreprises dont le siège se trouve au sein de l'Union européenne. Il existe donc une proximité certaine entre les deux types de dialogue social transnational d'entreprise. Pour autant, des différences subsistent entre les accords transnationaux d'entreprise ayant une vocation mondiale et ceux qui ont une vocation européenne, notamment en ce qui concerne les acteurs impliqués et les thématiques abordées, ce qui nous amène à les présenter de manière séparée.

\section{Au plan international}

On recense aujourd'hui une soixantaine d'accords transnationaux d'entreprise ayant vocation à s'appliquer dans le monde entier, et qui sont pour cette raison généralement appelés accords-cadres internationaux (pour une analyse approfondie de ces accords, voir SoBCZAK, 2007). Le nombre exact varie légèrement selon les études, dès lors que le champ d'application de ces accords n'est pas toujours bien précisé et qu'il y a une frontière flottante avec les accords ayant une vocation européenne seulement. L'immense majorité est signée par des entreprises dont le siège se trouve au sein de l'Union européenne, en particulier en Allemagne et en France (PAPADAKIS, 2008; SchommanN et al., 2000; SoBCZAK, 2006).

En l'absence d'un cadre juridique sur les accords transnationaux d'entreprise, de nombreuses questions se posent sur la légitimité des acteurs qui les négocient. En pratique (SOBCZAK, 2008), ces accords sont négociés et signés, d'un côté, par la direction de la société mère de la multinationale et, de l'autre, par une fédération syndicale internationale, accompagnée de plus en plus souvent par le comité d'entreprise européen (DA CostA, REHFELDT, 2009) et/ou

(3) Directive 94/45/CE du Conseil du 22 septembre 1994 concernant l'institution d'un comité d'entreprise européen ou d'une procédure dans les entreprises de dimension communautaire et les groupes d'entreprises de dimension communautaire en vue d'informer et de consulter les travailleurs.

(4) Directive 2001/86/CE, du Conseil du 8 octobre 2001, «complétant le statut de la Société européenne pour ce qui concerne l'implication des travailleurs». 
des organisations syndicales nationales du pays du siège. Aucun de ces acteurs ne s'est vu reconnaître un droit à la négociation collective transnationale par le droit du travail européen ou international.

Le contenu des accords transnationaux d'entreprise ayant un champ d'application mondial varie de manière importante, même si l'ensemble des accords sont signés par seulement un nombre restreint de fédérations syndicales internationales et si deux d'entre elles(5) ont élaboré des accords types constituant une base pour les négociations dans les entreprises. Presque tous les accords transnationaux d'entreprise contiennent l'engagement, de la part de l'entreprise, à respecter les droits fondamentaux au travail(6), en faisant explicitement référence aux conventions de l'Organisation internationale du travail dans ce domaine, ou à sa déclaration de 1998 relative aux principes et droits fondamentaux. Plus de la moitié de ces accords vont au-delà et contiennent également des dispositions sur les conditions de travail et d'emploi. Parmi les thématiques abordées, on trouve la protection de la santé et de la sécurité des salariés, le respect des normes nationales sur les salaires et le temps de travail, le droit à la formation et, plus rarement, l'anticipation et l'accompagnement social des restructurations. Enfin, un nombre plus restreint d'accords transnationaux d'entreprise, en particulier les plus récents, contiennent des engagements relevant de la responsabilité sociétale de l'entreprise et dépassent ainsi le champ traditionnel $\mathrm{du}$ droit du travail et de l'emploi, en abordant des thématiques comme la protection de l'environnement, l'investissement dans la communauté locale, le SIDA ou la lutte contre la corruption. Ils contribuent donc à relier les dimensions sociales, environnementales et économiques des activités des entreprises (COMMISSION EUROPÉENNE, 2008a et b; PAPADAKIs, 2008; SchoEmAnN et al., 2000).

\section{Au plan européen}

D'autres textes transnationaux négociés au sein des entreprises se limitent au territoire européen. Selon le document de travail que la Commission européenne a publié sur le sujet en juillet 2008, ces accords sont même plus nombreux (76) que ceux qui ont un champ d'application mondial (59). Si le statut juridique de ces deux catégories de textes ne diffère pas, chacune présente des spécificités quant aux acteurs impliqués et aux thématiques traités.

La grande majorité des accords transnationaux d'entreprise ayant un champ d'application européen sont évidemment négociés avec des entreprises

(5) La Fédération internationale des organisations de travailleurs de la métallurgie (FIOM) et l'Internationale des travailleurs du bâtiment et du bois (IBB).

(6) Interdiction de faire travailler des enfants, d'avoir recours au travail forcé, de procéder à des discriminations et la reconnaissance de la liberté syndicale et de négociation collective. dont le siège se trouve dans les États membres de l'Union européenne, la France accueillant à elle seule $34 \%$ de ces accords et $39 \%$ des entreprises les ayant signés (CoMmission eURopéEnNe, 2008b). Curieusement, on note cependant que les entreprises d'origine américaine ont aussi été particulièrement actives dans la négociation de tels accords à vocation européenne, ce qui contraste avec le fait que, parmi les entreprises ayant signé des accords à vocation mondiale, une seule est d'origine américaine. On peut sans doute l'expliquer par l'obligation relative à la directive européenne sur le comité d'entreprise européen à laquelle sont soumises les entreprises d'origine américaine d'une certaine taille(7).

Comme les accords à vocation mondiale, les accords transnationaux d'entreprise à vocation européenne sont signés par les dirigeants de la société mère du groupe. Selon la Commission euRopéEnNE (2008a), sur les quatre-vingt-neuf entreprises ayant signé un ou plusieurs accords, seules six ont signé à la fois un accord ayant un champ mondial ou mixte et un ou plusieurs accords européens. En ce qui concerne les secteurs dans lesquels des accords européens sont négociés, on peut constater, comme pour les accords mondiaux, la prédominance de la métallurgie. On peut cependant également noter que, dans certains secteurs, les entreprises se sont engagées davantage dans le dialogue social européen que dans le dialogue social international. C'est surtout le cas des entreprises du secteur de la finance, qui ont négocié très peu d'accords transnationaux à vocation mondiale, alors qu'elles sont très actives dans la négociation d'accords à vocation européenne. C'est aussi le cas, dans une moindre mesure, du secteur de l'alimentation.

Du côté des représentants des salariés, on retrouve les mêmes catégories d'acteurs pour les accords européens que pour les accords internationaux, bien que les fédérations européennes y remplacent en principe les fédérations internationales. En revanche, alors que les accords à vocation mondiale sont toujours signés par une fédération syndicale internationale, les fédérations syndicales européennes ne signent qu'un quart des accords transnationaux à vocation européenne. Ce sont les comités d'entreprise européens qui sont les plus actifs dans la signature de ces derniers. $77 \%$ des accords transnationaux à vocation européenne sont signés par un comité d'entreprise européen, dont encore les trois quarts par ce comité seul; $23 \%$ sont signés en commun avec une fédération syndicale européenne ou internationale; les $2 \%$ restants le sont en commun avec une fédération européenne et des organisations syndicales nationales.

(7) Article 4 de la directive 94/45/CE. 
Cette différence entre les signataires des accords transnationaux d'entreprise à vocation européenne et mondiale peut s'expliquer par le cadre juridique et institutionnel. Au plan international, aucune représentation des salariés n'est prévue par le droit du travail; la seule solution pour les entreprises souhaitant établir un dialogue social transnational à ce niveau consiste alors à négocier avec les fédérations syndicales sectorielles. Au plan européen, en revanche, avec le comité d'entreprise européen, il existe une institution de représentation des salariés qui peut constituer le cadre naturel de ce dialogue social transnational, même si la directive ne lui reconnaît pas ce droit et si la présence de représentants qui ne seraient pas désignés par les organisations syndicales peut poser des problèmes de reconnaissance et de légitimité.

Les thématiques abordées par les accords à vocation européenne diffèrent sensiblement de celles que traitent les accords à vocation mondiale. Les accords européens ont tendance à aborder des sujets plus spécifiques. La thématique la plus souvent abordée est celle des restructurations, certains accords visant à définir des principes généraux pour l'avenir, d'autres mettant en place des mesures d'accompagnement social dans le cadre d'une restructuration spécifique (PAPADAKIS, 2008; SchOEMANN et al., 2008). On peut aussi citer des accords qui portent sur la formation tout au long de la vie et qui visent à maintenir et renforcer l'employabilité des salariés, y compris dans le cas d'une restructuration future. Une autre thématique est celle du dialogue social et des procédures d'information et de consultation. D'autres accords à vocation européenne abordent des thèmes similaires à ceux des accords à vocation mondiale, comme la protection de la santé et de la sécurité, la responsabilité sociale de l'entreprise, les relations avec les sous-traitants ou encore l'égalité des chances. Enfin, certains accords à vocation européenne abordent des sujets techniques comme la protection des données ou l'intéressement et la participation des salariés.

\section{Le développement du dialogue social sectoriel européen : tendances récentes}

Contrairement aux négociations transnationales d'entreprises qui peuvent avoir un champ d'application européen ou mondial, le dialogue social transnational sectoriel reste limité au territoire de l'Union européenne, le secteur du travail maritime étant le seul exemple d'une négociation sectorielle au plan international (voir à ce sujet LILLIE, 2008).

Dans le contexte européen, le dialogue social sectoriel bénéficie d'un cadre juridique précis. Les articles 138 et 139 du traité CE, devenus depuis articles 154 et 155, reconnaissent l'importance du dialogue social européen au plan interprofessionnel et sectoriel et confèrent un statut juridique aux textes qui peuvent y être négociés. Les interlocuteurs sociaux européens se rencontrent surtout dans les comités de dialogue social dont le régime juridique est défini par une décision de la Commission du 20 mai 1998 (DufReSne et al., 2006).

Aujourd'hui, trente-six comités sectoriels sont reconnus, et d'autres sont encore en attente de reconnaissance(8). La participation à ces comités est conditionnée par trois critères de représentativité définis par la Commission européenne. Les organisations doivent «agir au niveau interprofessionnel ou appartenir à des secteurs ou catégories spécifiques et être organisées au niveau européen; être composées d'organisations elles-mêmes reconnues comme faisant partie intégrante des structures des partenaires sociaux des États membres, avoir la capacité de négocier des accords et être représentatives dans tous les États membres, dans la mesure du possible; disposer de structures adéquates leur permettant de participer de manière efficace au processus de consultation» (COM (93) 600 final du 14 décembre 1993). Du côté syndical, les membres des comités de dialogue social sont les fédérations syndicales européennes, qui sont membres de la Confédération européenne des syndicats. Les organisations patronales qui y participent ne sont en revanche pas membres de Business Europe (anciennement UNICE). Par ailleurs, alors que les mêmes fédérations syndicales participent souvent à plusieurs comités de dialogue social européen, du côté patronal, on trouve plutôt une fragmentation des acteurs.

En termes de thématiques traitées dans les comités sectoriels, les partenaires sociaux européens ont à assumer une double subsidiarité, à la fois horizontale et verticale. Horizontalement, il y a un lien fort entre les thématiques traitées dans le dialogue social européen et l'agenda de la Commission européenne (Pochet, 2006). Verticalement, les sujets traités doivent être pertinents pour l'ensemble, ou tout au moins la majorité, des États membres, mais sans empiéter sur les négociations nationales menées par les organisations membres (LÉONARD et al., 2007). En conséquence, si le champ que peut couvrir le dialogue social sectoriel européen peut théoriquement sembler très vaste, il est en pratique doublement cadré par le rôle des institutions européennes et par les interlocuteurs sociaux nationaux.

L'analyse que Pochet (Pochet et al., 2009) a effectuée sur les thématiques traitées montre qu'un premier groupe de sujets est en lien direct avec les politiques européennes en matière sociale, mais aussi en matière industrielle ou économique. Le thème du dialogue social est également très présent, notamment pendant la période entre 1999 et 2004, lorsque les comités ont rédigé un certain nombre de textes portant sur leur propre dialogue

(8) La liste complète des comités sectoriels est disponible sur le site de la Commission européenne: http://ec.europa.eu/ employment_social/social_dialogue/sectoral_fr.htm 
social et les élargissements de l'Union européenne. Par ailleurs, le dialogue social sectoriel européen couvre des thématiques classiquement traitées dans les relations collectives de travail comme la formation, la santé et la sécurité, les conditions de travail, l'emploi, le temps de travail ou, plus récemment, la diversité, la responsabilité sociale de l'entreprise ou le développement durable (Роснет et al., 2009).

\section{Quelles interactions entre les deux niveaux de dialogue social transnational?}

La question des interactions entre les accords transnationaux d'entreprise et les autres niveaux de dialogue social transnational n'a pas été directement traitée par la recherche à ce jour. Certes, le développement du dialogue social transnational s'est accompagné ces dernières années d'un nombre croissant de publications en droit, gestion ou sociologie. Ces recherches s'interrogent sur la contribution, actuelle ou potentielle, des relations professionnelles transnationales à la régulation sociale dans le contexte de la mondialisation (par exemple, BÉTHOux, 2006; Jobert, 2008; Moreau, 2006), sur l'intérêt d'un cadre juridique européen ou international dans ce domaine (Ales et al., 2006; Bourque, 2005; SobcZAK, 2007), ou sur les liens entre le dialogue social transnational et les relations professionnelles au plan national, régional ou local (MARGINSON, Sisson, 2004). D'autres travaux portent sur l'analyse, souvent comparative, d'expériences spécifiques de dialogue social transnational et leur impact sur les relations et les conditions de travail. Cependant, pour la plupart, les recherches se concentrent sur un niveau particulier, sans adopter une vision globale ou comparative des différents niveaux, ni chercher à explorer le potentiel ou la réalité de leurs interactions. Récemment, Mias (2009) explore les interactions multiples entre les textes européens et leur mise en œuvre au plan national, et en conclut qu'il existe une tendance à l'interpénétration des différents espaces qui, elle-même provient de l'interdépendance croissante de ces espaces. Toutefois, les interactions entre le dialogue social transnational au niveau de l'entreprise et au niveau sectoriel n'ont pas encore fait l'objet de recherches spécifiques à ce jour.

Ces interactions peuvent être envisagées de deux manières fondamentalement différentes:

- Le premier scénario est celui de la complémentarité et de l'articulation entre les deux niveaux. Les accords transnationaux d'entreprise comme les textes sectoriels européens s'inscriraient ainsi dans un système de dialogue social transnational à trois échelons, sur le modèle de certains systèmes nationaux, comportant le niveau interprofessionnel, sectoriel et de l'entreprise. Un tel système resterait nécessairement limité à l'Union européenne, dans laquelle le dialogue social est déjà institutionnalisé aux niveaux sectoriel et interprofessionnel. Dans ce scénario, se poserait la question de la répartition des rôles et des missions de chaque niveau et de la coordination verticale éventuelle entre les niveaux.

- Le deuxième scénario est celui de la concurrence entre les accords transnationaux d'entreprise et les textes sectoriels européens. Les accords transnationaux d'entreprise matérialiseraient alors, au plan transnational, une tendance générale à la décentralisation du dialogue social déjà observée dans les systèmes nationaux, au détriment des niveaux supérieurs que constituent l'interprofessionnel et le sectoriel (Ferner et Hyman, 1998; Traxler, 1995). Il faut toutefois signaler que décentralisation ne signifie pas nécessairement abandon de tout rôle de coordination par les niveaux supérieurs (Traxler, 1995 et 2003).

Si l'on compare les deux espaces de relations professionnelles transnationales, on note d'abord la différence quant au cadre juridique et institutionnel. À l'absence d'un cadre juridique et institutionnel relatif au niveau de l'entreprise répond le cadre institutionnel du dialogue social sectoriel européen clairement établi par les articles 154 et 155 (anciennement 138 et 139) du traité CE. Celui-ci se déroule au sein des comités institués et fortement soutenus par la Commission européenne, et sa structure et ses règles de fonctionnement sont établies dans des textes juridiques. Il réunit des partenaires sociaux dont la représentativité est reconnue selon des critères formels fixés par la Commission, et il produit des documents qui s'inscrivent dans une typologie des textes également définie par celle-ci. Au contraire, l'émergence et le développement des accords transnationaux d'entreprise ont pris place en l'absence d'un cadre juridique et institutionnel spécifique. Au-delà de ce constat, une comparaison plus fine permet toutefois d'identifier des similitudes et, plus encore, des liens, en termes d'acteurs, de thématiques et de processus de mise en œuvre des textes.

\section{Des acteurs multiples et en partie communs}

Les interactions entre les accords transnationaux d'entreprise et le dialogue social sectoriel européen pourraient se heurter à la stratégie d'acteurs syndicaux et patronaux qui tendent à privilégier chacun un niveau du dialogue social transnational. Analysant les actions des partenaires sociaux au niveau transnational, Marginson (in LÉONARD et al., 2007) souligne que les employeurs, dirigeants d'entreprises et fédérations patronales privilégient l'entreprise comme niveau d'action, alors que les organisations syndicales accordent la priorité au niveau sectoriel. Ce constat général conduirait alors à un développement parallèle des deux niveaux de dialogue social transnational sans favoriser leurs interactions, les acteurs patronaux cherchant à promouvoir les accords transnationaux d'entre- 
prise et les accords syndicaux se concentrant sur le dialogue social sectoriel européen.

La réalité est cependant plus nuancée. Ainsi, certaines fédérations syndicales européennes et internationales promeuvent très activement la conclusion d'accords transnationaux d'entreprise, notamment pour faire face à la réticence des acteurs patronaux à négocier des accords au niveau sectoriel. Par ailleurs, la conclusion d'un accord à quel que niveau que ce soit nécessite l'implication des deux partenaires sociaux. L'analyse des acteurs impliqués dans la négociation des accords transnationaux d'entreprise et le dialogue social sectoriel européen révèle en effet que certains de ces acteurs sont communs, pouvant ainsi favoriser des interactions et un enrichissement mutuel.

Cela se manifeste avant tout du côté des représentants des salariés. Du côté syndical, on trouve en effet les fédérations syndicales sectorielles aussi bien au niveau de l'entreprise que du secteur. Elles jouent un rôle clé dans la négociation et la mise en œuvre des accords transnationaux d'entreprise, qu'ils soient mondiaux ou européens. En particulier, la Fédération européenne des métallurgistes (FEM) et la Fédération européenne des syndicats des mines, de l'énergie et de la chimie (EMCEF), toutes deux membres de plusieurs comités de dialogue social sectoriel européen, s'impliquent aussi activement dans la négociation d'accords transnationaux d'entreprise, et surtout ceux à vocation européenne.

Le rôle actif de la FEM dans la négociation de ces accords s'inscrit clairement dans une stratégie mise en œuvre depuis le milieu des années 1990 pour tenter de coordonner les négociations collectives à travers les frontières en Europe. Comme le rappelle Pulignano (2010), l'objectif de la FEM en la matière consiste à éviter autant que possible une logique de concurrence entre les pays au risque d'une spirale négative en termes de pression sur les salaires et les conditions d'emploi. Cette stratégie se déploie au moyen de plusieurs dispositifs concrets comme la création de groupes de travail et comités comprenant des membres de la FEM ainsi que des représentants nationaux, l'organisation de conférences, définition de standards minimaux communs, l'établissement de règles de coordination des négociations dans les contextes nationaux ainsi que la mise en place de réseaux d'échange d'informations internes et partagé dès décembre 2006 avec le secteur textile et la chimie dans le portail «Eucob@n » (Pulignano, 2010; voir aussi FEM, 2006a, pour un historique détaillé de la stratégie de négociation collective coordonnée de la FEM). Cette stratégie de coordination des négociations collectives repose sur un engagement conjoint des membres nationaux de la FEM à adhérer aux lignes de conduite fixées au plan européen, et sur des principes communs de négociation, notamment en matière de formation continue (Pulignano, 2010). C'est donc bien dans ce cadre que s'inscrit l'établis- sement, en février 2006, d'une «procédure interne de la FEM pour les négociations au niveau des entreprises multinationales ». Cette procédure vise notamment à faire en sorte que la négociation d'un accord transnational d'entreprise implique une ou plusieurs organisations syndicales, et non pas le comité d'entreprise européen: «Il est dans l'intérêt de la FEM et de ses affiliés de garantir l'implication totale des syndicats dans tout processus de négociation potentiel, également au niveau européen » (FEM, 2006b, p. 1). La procédure porte sur les étapes de la négociation, non sur les thèmes qui y sont traités. Elle prévoit sept étapes que les membres nationaux s'engagent à respecter, portant notamment sur l'information et la négociation entre les syndicats impliqués dans l'entreprise, la FEM et le comité d'entreprise européen, la définition du mandat de négociation, l'élaboration et l'approbation du projet d'accord et, ensuite, la mise en œuvre.

Comme le souligne Bourque (2008), de telles tentatives de coordination transnationale par les fédérations syndicales européennes ne se font pas sans tensions avec les membres nationaux, dès lors qu'il s'agit pour ces derniers d'accepter de déléguer au plan européen l'établissement de lignes directrices qui vont, ensuite, limiter les marges de manœuvre des négociateurs au niveau national. Il faut également souligner qu'une telle stratégie est loin d'être présente dans toutes les fédérations syndicales européennes.

Du côté patronal, les acteurs du dialogue social sectoriel européen et de la négociation d'accords transnationaux d'entreprise sont en principe différents. Alors que les premiers impliquent les fédérations patronales européennes reconnues comme représentatives, les accords transnationaux d'entreprise sont négociés par la direction d'une entreprise donnée. Ce constat peut cependant être nuancé pour les secteurs dans lesquels ce sont des entreprises qui représentent directement les membres nationaux des organisations européennes d'employeurs. C'est le cas des secteurs dans lesquels des entreprises publiques bénéficiaient dans le passé d'un monopole comme, par exemple, les services postaux ou l'électricité. Dans ces cas, les mêmes acteurs patronaux peuvent se trouver impliqués aussi bien dans le dialogue social sectoriel européen et dans la négociation d'accords transnationaux d'entreprise(9).

Ainsi, EDF, signataire d'un accord transnational à vocation mondiale, est aussi très impliquée dans le dialogue social sectoriel européen, car elle représente la France au sein du comité sectoriel de l'électricité.

(9) On peut se demander si cette situation perdurera avec l'ouverture de ces secteurs à la concurrence qui conduira à une représentation plus éclatée dans les associations d'employeurs, risquant ainsi de freiner le développement du dialogue social sectoriel au moment même où son importance s'accroît en tant que régulateur des conditions sociales de la concurrence. 
Si les personnes de l'entreprise qui participent aux différents processus ne sont pas les mêmes, elles se coordonnent néanmoins entre elles et favorisent les interactions entre les accords transnationaux d'entreprise et les activités du comité de dialogue social sectoriel européen. Ainsi, la responsabilité sociale, thème central de l'accord mondial négocié au sein $\mathrm{du}$ groupe EDF, est également devenu un sujet de dialogue au sein du comité de dialogue social européen de l'électricité. La responsabilité sociale des entreprises a en effet fait l'objet d'un texte sectoriel conjoint en décembre 2004 (EMCEF, EURELECTRIC, Epsu, 2004) et figure dans le programme de travail du comité de dialogue social européen de l'électricité depuis plusieurs années (Pochet et al., 2009). En novembre 2006, un atelier a été organisé sur le sujet dans le cadre du comité de dialogue social sectoriel européen, permettant aux représentants d'EDF de partager leur expérience sur la pertinence de faire de la responsabilité sociale un enjeu du dialogue social. En revanche, la fédération européenne patronale Eurelectric affirme fortement sa réticence à entrer dans un processus de négociation dans le cadre du comité de dialogue social européen.

\section{Les thématiques: \\ une "convergence sans coordination"}

Les interactions entre les accords transnationaux d'entreprise et le dialogue social sectoriel européen peuvent aussi s'analyser du point de vue des thématiques abordées. Il s'agit dès lors de vérifier si ces thèmes sont les mêmes aux deux niveaux de dialogue social transnational, et s'ils y sont traités avec le même degré de précision. La comparaison n'est pas aisée à établir, car les classifications utilisées pour désigner les thématiques varient, et parce qu'un même texte peut couvrir plusieurs thèmes. Il faut aussi relativiser la solidité des données disponibles, dès lors qu'elles rendent compte exclusivement des thèmes présents dans les textes signés par les parties, et non pas des sujets discutés mais non formalisés dans des textes conjoints. Néanmoins, l'analyse exploratoire des sujets abordés dans les accords transnationaux d'entreprise et les textes issus du dialogue social sectoriel européen révèle que certaines thématiques sont spécifiques à chacun des deux niveaux alors que d'autres sont communs (tableau 1).

Tableau 1 : Présence des thèmes dans les textes

\begin{tabular}{|c|c|c|c|}
\hline Thème & $\begin{array}{c}\text { Accords } \\
\text { d'entreprises } \\
\text { mondiaux }\end{array}$ & $\begin{array}{l}\text { Accords d'en- } \\
\text { treprises euro- } \\
\text { péens }\end{array}$ & $\begin{array}{c}\text { Textes issus du } \\
\text { dialogue social } \\
\text { sectoriel européen } \\
(1999-2007) \\
\end{array}$ \\
\hline Aspects sociaux des politiques de l'Union européenne & & & $\mathrm{x}$ \\
\hline Conditions de travail & $\mathrm{x}$ & $\mathrm{x}$ & $\mathrm{x}$ \\
\hline Développement durable, protection de l'environnement & $\mathrm{x}$ & & $\mathrm{x}$ \\
\hline Dialogue social & $\mathrm{x}$ & $\mathrm{x}$ & $\mathrm{x}$ \\
\hline Égalité des chances, non-discrimination & $\mathrm{x}$ & $\mathrm{x}$ & $\mathrm{x}$ \\
\hline Élargissement de l'Union européenne & & & $\mathrm{x}$ \\
\hline Emploi & $\mathrm{x}$ & $\mathrm{x}$ & $\mathrm{x}$ \\
\hline Formation & $\mathrm{x}$ & $\mathrm{x}$ & $\mathrm{x}$ \\
\hline Interdiction du travail des enfants & $\mathrm{x}$ & & \\
\hline Interdiction du travail forcé & $\mathrm{x}$ & & \\
\hline Liberté syndicale & $\mathrm{x}$ & & \\
\hline Participation financière & & $\mathrm{x}$ & \\
\hline Politiques économiques et sociales & & & $\mathrm{x}$ \\
\hline Protection des données & & $\mathrm{x}$ & \\
\hline Rémunération & $\mathrm{x}$ & & \\
\hline Restructurations & $\mathrm{x}$ & $\mathrm{x}$ & \\
\hline Responsabilité sociale de l'entreprise & $\mathrm{x}$ & $\mathrm{x}$ & $\mathrm{x}$ \\
\hline Santé et sécurité & $\mathrm{x}$ & $\mathrm{x}$ & $\mathrm{x}$ \\
\hline SIDA & $\mathrm{x}$ & & \\
\hline Sous-traitance & $\mathrm{x}$ & $\mathrm{x}$ & \\
\hline Temps de travail & $\mathrm{x}$ & & $\mathrm{x}$ \\
\hline
\end{tabular}

Sources des données: Commission européenne, 2008b pour les accords transnationaux d'entreprise; Pochet et al., 2009 pour les textes du dialogue social sectoriel européen. 
Le tableau 1 montre que les textes sectoriels européens portent sur des thématiques en lien direct avec l'intégration européenne ou les activités et politiques de l'Union européenne, comme les aspects sociaux des politiques européennes, les enjeux de l'élargissement, les politiques d'emploi ou plus largement les politiques économiques et sociales. Au contraire, d'autres thématiques sont spécifiques aux accords transnationaux d'entreprises et ne figurent pas, ou très peu, dans les textes issus du dialogue social sectoriel européen. C'est le cas surtout des droits fondamentaux au travail, une thématique qui figure dans presque tous les accords transnationaux d'entreprise à vocation mondiale, mais qui n'est que rarement abordée dans le dialogue social sectoriel européen. De même, l'anticipation et la gestion responsable des restructurations sont des sujets prioritaires des accords transnationaux d'entreprise à vocation européenne et sont bien moins abordés dans le dialogue social sectoriel européen. Il en va de même des thématiques spécifiques traitées dans certains accords transnationaux d'entreprise à vocation européenne, comme la participation financière ou la protection des données.

À côté de cela, un grand nombre de sujets sont communs aux deux niveaux. Des thèmes comme les conditions de travail, la santé et la sécurité, l'emploi, la formation, l'égalité des chances, la responsabilité sociale ou le développement durable sont en effet traités aussi bien dans des accords transnationaux d'entreprise que dans des textes issus du dialogue social sectoriel européen.

Le simple fait que ces thématiques sont communes aux deux niveaux du dialogue social transnational ne permet cependant pas de déduire qu'il y ait une réelle coordination entre ces deux niveaux visant, par exemple, à décliner par des accords transnationaux d'entreprise une thématique préalablement explorée dans le cadre du dialogue social sectoriel européen ou, au contraire, à encadrer par des accords au niveau sectoriel des thématiques figurant dans plusieurs accords transnationaux d'entreprise. On peut plutôt conclure à ce que HancKé (2002) appelle une "convergence sans coordination», par laquelle, face à des problèmes comparables, des solutions similaires sont développées aux différents niveaux de dialogue social transnational sans que cela traduise une coordination organisée.

Une analyse plus approfondie des textes négociés aux deux niveaux de dialogue social transnational fait apparaître des différences importantes en termes de précision sur les thèmes communs. Le plus souvent, le niveau de l'entreprise se caractérise par un degré de précision plus important et des mesures de mises en œuvre plus détaillées, mais ce n'est pas toujours le cas. Certains textes négociés dans le cadre du dialogue social sectoriel européen définissent par exemple des règles très précises et mettent en place des procédures de suivi très détaillées.

\section{La mise en œuvre: des procédures similaires malgré des cadres juridiques différents}

L'analyse des accords transnationaux d'entreprise et des textes négociés dans le dialogue social sectoriel européen révèle que, malgré des cadres juridiques et institutionnels différents, des procédures similaires sont parfois mobilisées pour assurer la mise en œuvre de ces textes.

Dans le cadre du dialogue social sectoriel européen, la Commission européenne a élaboré une typologie des différents textes négociés entre les partenaires sociaux, en distinguant selon les procédures de mise en œuvre (CEC, 2004). Une première catégorie de textes comprend des «accords» qui se caractérisent par l'existence d'une obligation de mise en œuvre à une date précise. Cette catégorie comprend deux types d'accords prévus par l'article 139 du traité : les accords mis en œuvre par une décision du Conseil et les accords dits autonomes mis en œuvre par les partenaires sociaux dans chaque État membre. Une deuxième catégorie comporte des textes fondés sur les processus comme des «cadres d'action», des "lignes directrices», des «codes de conduite» ou des « orientations politiques» qui contiennent des recommandations aux membres nationaux pour qu'un suivi et une évaluation régulière de la mise en œuvre soient effectués. Les «avis conjoints» et les «déclarations» constituent une troisième catégorie de textes qui ont une fonction d'information et ne prévoient pas de mécanisme particulier de mise en œuvre. Dans la quatrième catégorie, on trouve des textes procéduraux qui établissent des règles pour le dialogue entre les partenaires sociaux. Enfin, une dernière catégorie de textes est constituée de rapports de suivi par lesquels les partenaires sociaux établissent conjointement un bilan sur la mise en œuvre d'un texte relevant d'une des autres catégories.

Quelle que soit la nature du texte, les membres des comités de dialogue social sectoriel européen sont, en pratique, confrontés à une incertitude quant à la mise en œuvre des accords. D'une part, les partenaires sociaux européens ne disposent pas de pouvoir de contrainte sur leurs membres nationaux chargés de transposer les textes dans chaque État membre. D'autre part, la mise en œuvre d'un texte transnational requiert à la fois sa traduction, sa transposition et sa mise en pratique (KelLer, 2003). Or, même la question de la traduction peut s'avérer difficile dans la mesure où elle dépend fortement des institutions et des dynamiques internes à chaque pays. De même, l'évaluation de la mise en œuvre est un exercice méthodologique complexe qui suppose la coopération active de la part des membres nationaux pour fournir des données fiables aux partenaires sociaux européens. Les recherches récentes sur le sujet montrent donc que les partenaires sociaux européens eux-mêmes rencontrent des 
difficultés pour évaluer dans quelle mesure un texte est mis en œuvre dans un secteur donné au sein des différents États membres (PoCHET et al., 2009). Les acteurs reconnaissent manquer d'outils qui permettraient une évaluation systématique de la mise en œuvre, tout en observant qu'une telle évaluation est essentielle pour assurer la crédibilité du dialogue social européen (LÉONARD et al., 2007). Pour faire face à ces enjeux, les partenaires sociaux européens ont ainsi progressivement développé une gamme de différents outils destinés à assurer le suivi de la mise en œuvre de leurs textes qui complètent les dispositifs juridiques. Ils mènent des enquêtes, établissent des groupes de travail, organisent des conférences, publient des rapports ou créent des sites Internet (Pochet et al., 2009).

En l'absence d'un cadre juridique pour le dialogue social transnational au sein des entreprises, aucune typologie n'a été établie à ce jour pour distinguer les différents accords négociés à ce niveau en fonction des procédures de mise en œuvre. L'analyse de ces accords révèle cependant que les signataires créent des procédures similaires à celles qui existent au niveau sectoriel. Presque tous les accords transnationaux d'entreprise contiennent des dispositions sur la mise en œuvre et prévoient au moins l'organisation d'une réunion annuelle entre les signataires pour effectuer un bilan des actions engagées et des résultats obtenus. Plusieurs accords créent des procédures de résolution des conflits permettant aux salariés de dénoncer le non-respect des droits reconnus par les accords sans saisir une juridiction étatique. En principe, le salarié est invité à saisir ses supérieurs hiérarchiques, éventuellement avec le soutien des représentants syndicaux locaux. Si le problème ne peut être résolu à ce niveau, il est porté à la connaissance des représentants de la direction et des salariés au niveau national, et, en cas de nouvel échec, à celle des signataires de l'accord au niveau transnational. Ce type de procédure crée un système d'alerte interne permettant aux dirigeants d'identifier d'éventuelles violations des accords et, le cas échéant, d'y remédier sans faire appel ni aux médias, ni aux juridictions étatiques.

Certains accords transnationaux d'entreprise vont plus loin et prévoient leur déclinaison par la négociation d'accords entre les partenaires sociaux dans les différentes filiales. Intégrées dans les accords conclus au sein d'EDF et de PSA Peugeot Citroën, ces procédures sont inspirées des accords autonomes dont le régime est fixé par l'article 139 du traité pour les accords au niveau sectoriel ou interprofessionnel. Elles peuvent permettre de stimuler le dialogue social dans les différentes entités de l'entreprise dans le monde et d'adapter le contenu général des accords transnationaux aux spécificités locales. Enfin, compte tenu du pouvoir hiérarchique dont disposent les dirigeants du siège sur les dirigeants des filiales, et qui fait défaut aux partenaires sociaux européens aussi bien du côté patronal que du côté syndical, la mise en œuvre des accords transnationaux d'entreprise peut aussi se traduire par l'établissement de processus managériaux. Ainsi, après consultation des signataires syndicaux, les dirigeants du groupe EDF ont défini des indicateurs de suivi de l'accord transnational et mis en place un processus de reporting obligatoire pour les dirigeants de toutes les filiales.

Comme nous l'avons indiqué pour les thématiques communes, rien ne prouve que l'existence de procédures de mise en œuvre similaires dans les accords transnationaux d'entreprise et les textes issus du dialogue social sectoriel européen traduit une réelle coordination entre ces deux niveaux de dialogue social transnational. D'ailleurs, à ce jour les partenaires sociaux n'envisagent pas les accords transnationaux d'entreprise comme l'un des moyens de mettre en œuvre les textes négociés dans le cadre du dialogue social sectoriel européen. Les similitudes dans les procédures de suivi et de mise en œuvre des deux types de textes semblent donc bien davantage liées à l'existence de problématiques similaires quant à la transposition locale des accords transnationaux, voire parfois à l'implication des mêmes acteurs dans la négociation des deux types de textes.

\section{$*$ \\ $* \quad *$}

La comparaison entre les accords transnationaux d'entreprise et le dialogue social sectoriel européen fait apparaître certaines différences, la plus importante étant celle du cadre juridique et institutionnel. Cela pourrait inciter à conclure à l'existence de deux espaces spécifiques, étrangers l'un à l'autre. L'analyse permet toutefois de constater qu'il y a non seulement des "recouvrements partiels» (JogODZINSKI, 2007), mais aussi une certaine «porosité» (DAUGareiLh, 2008) et des «influences réciproques» (MIAS, 2009) entre les deux niveaux.

Certes, le dialogue social européen sectoriel prend appui sur des fondements institutionnels clairs établis dans le traité, alors que les accords transnationaux d'entreprise se développent en dehors de tout cadre juridique et institutionnel. Mais deux éléments amènent à relativiser cette opposition. D'un côté, l'enjeu au niveau sectoriel n'est pas tant la traduction des fondements institutionnels que la construction d'un espace européen de négociation collective fondé sur des acteurs prêts à négocier et à mettre en œuvre des textes conjoints (DUFRESNE et al., 2006; Pochet et al., 2009). De l'autre côté, les accords d'entreprise sont, plus qu'il ne semble au premier abord, liés à l'intégration européenne, par leur portée, par leurs acteurs et par leurs préoccupations au sein de l'espace commun européen. 
Ensuite, le dialogue social transnational en entreprise et le dialogue social sectoriel européen reflètent des stratégies différentes de la part des acteurs dans les deux espaces: les acteurs patronaux tendent à privilégier le niveau de l'entreprise; les acteurs syndicaux tendent à privilégier le niveau sectoriel (MARGINSON, in LÉONARD et al., 2007). Toutefois des acteurs communs agissent dans les deux espaces. Du côté syndical, c'est surtout vrai lorsque la FEM intervient, dans le cadre d'une stratégie volontariste de coordination de la négociation collective au travers des frontières et au plan sectoriel. Ici, il s'agit bien de coordination, et non plus de simple convergence. Cela reste cependant marginal si l'on considère le petit nombre de textes d'entreprise impliquant une fédération syndicale européenne, et le fait que seules la FEM et l'EMCEF y participent à ce jour aux côtés d'une diversité d'autres signataires. Les thèmes traités se recouvrent aussi partiellement, ce qui reflète des préoccupations communes, mais résulte également de mécanismes de diffusion que portent certains acteurs. Quant à la mise en œuvre des textes, on trouve de part et d'autre des préoccupations similaires et une expérimentation de pratiques, même si le dialogue social sectoriel européen s'inscrit dans un cadre nettement plus formalisé.

En somme, si l'on se réfère uniquement à des stratégies d'acteurs qui préfèrent investir en priorité un lieu plutôt qu'un autre, on doit bien conclure à une concurrence des modèles. Toutefois, il faut dépasser ce premier constat, dans la mesure où la «porosité» existe bel et bien et ouvre des pistes à une complémentarité dans le contexte de l'Union européenne. Les développements récents dans les deux espaces confirment l'engagement des interlocuteurs sociaux au plan transnational dans des formes souples de régulation aussi bien au niveau du secteur que de celui de l'entreprise. En ce sens, les accords transnationaux d'entreprise ne constituent pas un phénomène «à part», qui serait parfaitement spécifique et séparé des logiques interprofessionnelles et sectorielles au plan européen. Ils participent eux aussi au déploiement de formes de dialogue social non contraignant identifié par GoETSCHY (2005).

Il ne s'agit pas, à ce stade, d'une architecture institutionnalisée de relations collectives transnationales, et chaque niveau répond à des logiques qui lui sont propres mais, en même temps, les acteurs investissent ces différents espaces tout en assurant un certain nombre d'interactions qui montrent, à tout le moins, que les niveaux sont interdépendants au sein d'un contexte européen où les enjeux économiques et sociaux dans les États membres sont euxmêmes de plus en plus enchevêtrés au travers des frontières. 


\section{Bibliographie}

Ales E., Engblom S., Jaspers T., Laulom S., Sciarra S., SobczaK A. \& VAldés DAL-Ré F. (2006), Transnational collective bargaining: past, present and future, Brussels, European Commission.

BÉTHoux E. (2006), Entreprises multinationales et représentation des salariés en Europe. L'expérience des comités d'entreprise européens, thèse de sociologie sous la direction de Jobert A., IDHE, université de Paris X-Nanterre, soutenance le 8 décembre 2006.

BÉTHoux E. (2008), «Le dialogue social transnational dans l'entreprise: dynamiques européennes », in A. Jobert (sous la direction de), Les nouveaux cadres $d u$ dialogue social, Bruxelles, Peter Lang, pp. 189-246.

BouRque R. (2005), Les accords-cadres internationaux (ACI) et la négociation collective internationale à l'ère de la mondialisation, Genève, Institut international d'études sociales (DP/161/2005).

Bourque R. (2008), “International framework agreements and the future of collective bargaining in multinational companies", Just Labour: A Canadian Journal of Work and Society, Vol. 12, spring 2008, pp. 30-47.

Commission européenne (1998), Commission Decision 98/500/EC of 20 May 1998 on the Establishment of Sectoral Dialogue Committees Promoting the Dialogue between the Social Partners at European Level, OJ L 225 of 12 August 1998, pp. 0027-0028.

COMmission EuRopéEnNe (2004), Communication from the Commission "Partnership for change in an enlarged Europe - Enhancing the contribution of European social dialogue”, COM (2004) 557 final.

Commission EURopéEnNe (2008a), Mapping of transnational texts negotiated at corporate level, Brussels, European Commission, EMPL F2 EP/bp 2008 (D) 14511.

Commission européEnNe (2008b), Document des services de la Commission. Le rôle des accords d'entreprise transnationaux dans le contexte d'une intégration internationale croissante, Bruxelles, Commission des Communautés européennes, SEC (2008) 2155.

Da Costa I., Rehfeldt U. (2009), «Les CEE et la négociation collective transnationale: les accords européens et mondiaux dans l'automobile», Revue de l'IRES, $\mathrm{n}^{\circ}$ 61, pp. 99-127.

Daugareilh I. (2008), «Les fonctions de la négociation transnationale d'entreprise», communication à la conférence «Accords transnationaux d'entreprise; dialogue, droits, anticipation des restructurations, acteurs: une nouvelle perspective», conférence organisée par la Présidence française de l'Union européenne, Lyon, 13-14 novembre 2008 .

Didry C., Mias A. (2005), Le moment Delors. Les syndicats au cour de l'Europe sociale, Bruxelles, Peter Lang.
Dufresne A., Degryse C. \& Pochet P. (2006), The European sectoral social dialogue. Actors, developments and challenges, Bruxelles, Peter Lang.

Emcef, Eurelectric, Epsu (2004), Corporate social responsibility and the European electricity sector, Brussels, December.

FEM (2006a), Milestones - Jalons - Meilensteine, Bruxelles, Fédération européenne des métallurgistes, http:// www.eucoban.eu/EMF/EMF-Collective-BargainingPolicy/Publications/Milestones

FEM (2006b), Procédure interne de la FEM pour les négociations au niveau des entreprises multinationales, Bruxelles, Fédération européenne des métallurgistes.

Ferner A., Hyman R. (1998), "Introduction: Towards European industrial relations?" in Ferner A., Hyman R. (eds.), Changing industrial relations in Europe, 2nd edition, Oxford, Blackwell, pp. XI-XXVI.

Goetschy J. (2005), “The European social dialogue in the 1990s : institutional innovations and new paradigms", Transfer, Vol. 11, n 3, pp. 409-423.

HANCKÉ B. (2002), “The political economy of wage-setting in the Eurozone", in P. Pochet (ed.), Wage policy in the Eurozone, Brussels, Peter Lang, pp. 131-148.

Jobert A. (2008), Les nouveaux cadres du dialogue social. Europe et territoires, Bruxelles, Peter Lang.

Keller B. (2003), "Social dialogue at Sectoral Level: The Neglected Ingredient of European Industrial Relations", in B. Keller \& H.W. Platzer, (eds.), Industrial Relations and European Industrial Integration: Transand Supranational Developments and Prospects, Ashgate, Aldershot, pp. 30-57.

Léonard E., Erne R., Marginson P., Smismans S. (2007), New structures, forms and processes of governance in European industrial relations, Research report for the European Foundation for the Improvement of Living and Working Conditions, Luxembourg, Office for Official Publications of the European Communities.

Lillie N. (2008), "The ILO Maritime Labour Convention, 2006: A new paradigm for global labour rights implementation" in K. Papadakis (ed.) Cross-border social dialogue and agreements, An emerging global industrial relations framework? Geneva, International Labour Office, International Institute for Labour Studies, pp. 191-218.

Marginson P., Sisson K. (2004), Europeanisation, Integration and Industrial Relations. Multilevel Governance in the Making, Basingstoke, Palgrave Macmillan.

MiAs A. (2004), «Du dialogue social européen au travail legislative communautaire: Maastricht, ou le syndical saisi par le politique», Droit et Société, n 58, pp. 657680 . 
Mias A. (2009) Le dialogue social européen en interactions: influences et interférences des différents instruments de l'Europe sociale sur les politiques et pratiques aux différents niveaux, de l'Europe à l'entreprise, document de travail, Paris, Fondation Europe et Société.

Moreau M.-A. (sous la direction de) (2006), Normes sociales, droit du travail et mondialisation, Paris, Dalloz.

PAPADAKIS K. (ed.) (2008), Cross-Border Social Dialogue and Agreements : An emerging global industrial relations framework?, Geneva, International Institute for Labour Studies/International Labour Organisation.

Pochet P. (2006), “A Quantitative Analysis”, in Dufresne A., Degryse C. and Pochet, P. (eds.), The European Sectoral Social Dialogue. Actors, Developments and Challenges, Brussels, Peter Lang, pp. 83-108.

Pochet P., Peeters A., Léonard E. \& Perin E. (2009), Dynamics of the European sectoral social dialogue. Report to the European Foundation for the Improvement of Living and Working Conditions, Luxembourg, Office for Official Publications of the European Communities (forthcoming).

Pulignano V. (2010), «Syndicats et coordination des négociations collectives en Europe», in E. Arcq, M. Capron, E. Léonard \& P. Reman (eds) Dynamiques de la concertation sociale, Bruxelles, CRISP (sous presse).
Schoemann I., Sobczak A., Voss E. \& Wilke P. (2008), Codes of conduct and international framework agreements: New forms of governance at company level, Report to the European Foundation for the Improvement of Living and Working Conditions, Luxembourg, Office for Official Publications of the European Communities.

Sobczak A. (2006), «Les accords-cadre internationaux: un modèle pour la négociation collective transnationale?», Oeconomia Humana, vol. 4(4), pp. 13-18.

SobczaK A. (2007), "Legal Dimensions of International Framework Agreements in the Field of Corporate Social Responsibility", Relations Industrielles/Industrial Relations, Vol. 62(3), pp. 466-491.

SobczaK A. (2008), «Syndicats et responsabilité sociale des multinationales », Gestion - Revue internationale de Gestion, vol. 33(1), pp. 18-26.

TraXler F. (1995), "Farewell to Labour Market Associations? Organized versus Disorganized Decentralization as a Map for Industrial Relations", in C. Crouch, F. Traxler (eds), Organized Industrial Relations in Europe: What Future?, Aldershot, Avebury, pp. 23-44.

TRAXler F. (2003), "Coordinated bargaining: a stocktaking of its preconditions, practices and performance", Industrial Relations Journal, 34 :3, pp. 194-209. 